\title{
Neuropsychological, Neurovirological and Neuroimmune Aspects of Abnormal GABAergic Transmission in HIV Infection
}

\author{
Tetyana Buzhdygan ${ }^{1,2}$ - Joshua Lisinicchia ${ }^{1}$ - Vipulkumar Patel ${ }^{1}$ - Kenneth Johnson ${ }^{3}$. \\ Volker Neugebauer $^{4}$ • Slobodan Paessler ${ }^{1}$ - Kristofer Jennings ${ }^{5}$ • Benjamin Gelman ${ }^{1,2}$
}

Received: 19 August 2015 / Accepted: 18 January 2016 / Published online: 30 January 2016

(C) The Author(s) 2016. This article is published with open access at Springerlink.com

\begin{abstract}
The prevalence of HIV-associated neurocognitive disorders (HAND) remains high in patients with effective suppression of virus replication by combination antiretroviral therapy (cART). Several neurotransmitter systems were reported to be abnormal in HIV-infected patients, including the inhibitory GABAergic system, which mediates fine-tuning of neuronal processing and plays an essential role in cognitive functioning. To elucidate the role of abnormal GABAergic transmission in HAND, the expression of GABAergic markers was measured in 449 human brain specimens from HIV-infected patients with and without HAND. Using realtime polymerase chain reaction, immunoblotting and immunohistochemistry we found that the GABAergic markers were
\end{abstract}

Electronic supplementary material The online version of this article (doi:10.1007/s11481-016-9652-2) contains supplementary material, which is available to authorized users.

Benjamin Gelman

bgelman@utmb.edu

1 Department of Pathology, University of Texas Medical Branch, 301 University Blvd, 77555-0609 Galveston, TX, USA

2 Department of Neuroscience and Cell Biology, University of Texas Medical Branch, 301 University Blvd, 77555-0609 Galveston, TX, USA

3 Department of Pharmacology and Toxicology, University of Texas Medical Branch, 301 University Blvd, 77555-0609 Galveston, TX, USA

4 Department of Pharmacology and Neuroscience, School of Medicine, Texas Tech University Health Sciences Center, 3601 4th Street, 79430-6592 Lubbock, TX, USA

5 Department of Preventive Medicine and Community Health, University of Texas Medical Branch, 301 University Blvd, 77555-0609 Galveston, TX, USA significantly decreased in most sectors of cerebral neocortex, the neostriatum, and the cerebellum of HIV-infected subjects. Low GABAergic expression in frontal neocortex was correlated significantly with high expression of endothelial cell markers, dopamine receptor type 2 (DRD2L), and preproenkephalin (PENK) mRNAs, and with worse performance on tasks of verbal fluency. Significant associations were not found between low GABAergic mRNAs and HIV1 RNA concentration in the brain, the history of cART, or HIV encephalitis. Pathological evidence of neurodegeneration of the affected GABAergic neurons was not present. We conclude that abnormally low expression of GABAergic markers is prevalent in HIV-1 infected patients. Interrelationships with other neurotransmitter systems including dopaminergic transmission and with endothelial cell markers lend added support to suggestions that synaptic plasticity and cerebrovascular anomalies are involved with HAND in virally suppressed patients.

Keywords Autopsy · Glutamate decarboxylase - GABA · GABAergic $\cdot$ HIV associated neurocognitive disorders $\cdot$ HIV encephalitis

\section{Introduction}

The prevalence of HIV-associated neurocognitive disorders (HAND) is substantial in patients treated with cART (McArthur et al. 2010). HAND was linked to the neuropathological changes produced by HIV encephalitis (HIVE) before the era of cART (Budka 1991). Data from the cART era, however, are far less supportive of the putative association between HAND and HIVE (Gelman 2015). While the frequency of HIVE declined in cART-treated patient cohorts to below $5 \%$, the prevalence of HAND remains as high as $50 \%$ 
(McArthur et al. 2010). Neurovirological and brain gene expression data both show that the overwhelming majority of patients with HAND taking cART do not have HIVE, and they are not likely to harbor a high concentration of replicating HIV in the CNS (Gelman et al. 2012a, 2013). Thus, the pathophysiology of HAND remains poorly understood, especially when virus replication is suppressed with cART.

HIVE is associated with synaptodendritic simplification and neuronal loss in the later stages of infection (Masliah et al. 1997). Concentrations of several synaptic structural proteins are abnormal and local protein turnover in synapses may be abnormal in HIVE (Nguyen et al. 2010; Gelman and Nguyen 2010). Those aspects of the neuropathology imply that cART-treated patients might harbor subtle types of dysfunction in synaptic transmission due to synaptic plasticity, or functional changes that are not driven by morphological changes or neurodegeneration (Gelman et al. 2004, 2012b). There is neurochemical evidence that GABAergic (Koutsilieri et al. 2001; Gelman et al. 2012b), dopaminergic (Nath et al. 2000; Koutsilieri et al. 2001; Gelman et al. 2006; Gelman et al. 2012a, 2012b), cholinergic (Koutsilieri et al. 2001), glutamatergic (Ernst et al. 2010) and serotonergic (Murray 2003) neurotransmitter systems are perturbed in these patients. Decreased immunostaining of GABAergic neocortical neurons in HIVE is especially well-documented (Masliah et al. 1992, 1995; Fox et al. 1997). GABAergic markers including calbindin and somatostatin exhibit decreased immunostaining in tissue sections with HIVE (Masliah et al. 1992, 1995; Fox et al. 1997), and potential synergy between HAND and concomitant methamphetamine abuse has been suggested for these outcomes (Langford et al. 2003; Chana et al. 2006). Other evidence shows that several mRNA transcripts pertaining to GABAergic proteins are dysregulated in the dorsolateral prefrontal cortex (DLPFC) of HIVE patients (Gelman et al. 2004, 2012a). Whether disturbances in neural transmission in HIV infection represent synaptic plasticity among viable synaptic networks, versus outright neuronal death and subsequent degeneration remains controversial.

Inhibitory GABAergic interneurons constitute $20 \%$ of the total number of neurons in the neocortex. These neurons modulate the activity of principal cells via alteration of the synchronization of excitatory activity of pyramidal neurons, and this is associated with cognitive and behavioral functions (Markram et al. 2004). Abnormal GABAergic neural transmission is present in brain specimens from patients with epilepsy, major depressive disorders and schizophrenia (Prince and Wilder 1967; Sanacora and Saricicek 2007; Hashimoto et al. 2008). Neurocognitive impairment in these diseases is driven in part by abnormal output of the DLPFC, and can lead to worse performance on tasks of abstract/executive function and verbal fluency (Volk et al. 2000; Thompson et al. 2009). The same underlying neuropsychological disorders often occur in patients with HAND (Klinkenberg et al. 2004; Iudicello et al.
2007) and could worsen and/or contribute to dysfunction of the DLPFC in patients with HAND (Woods et al. 2009).

In addition to modulating neocortical output, GABAergic interneurons play a pivotal role in neurovascular coupling, in which neurotransmitters regulate local microvascular blood flow in the neurovascular unit (Fergus and Lee 1997; Kocharyan et al. 2008). Using blood oxygen dependent contrast imaging (BOLD), several diseases exhibit disturbed regulation of cerebral blood flow, which often is linked to changes in GABAergic transmission (Northoff et al. 2007). Cerebral blood flow assessed using BOLD also is abnormal in HIV infected patients with and without HAND; the mechanism is not known and a potential role for altered GABAergic transmission has not been examined heretofore (Ances et al. 2011; Towgood et al. 2012). Thus, altered cerebral blood flow might be involved in HAND and HIV-associated changes in neurovascular coupling.

GABAergic neurons synthesize GABA via a rate-limiting reaction that is catalyzed by two glutamic acid decarboxylase (GAD) enzymes (GAD67 and GAD65, respectively). These two proteins are encoded by independently regulated genes located on chromosomes 2 and 10 (GAD1 and GAD2, respectively). Both proteins are useful histological markers of GABAergic interneurons, although they exhibit unique patterns of cellular expression (Soghomonian and Martin 1998). Another marker of GABAergic interneurons is the gap junction protein connexin 36 (encoded by GJD2 mRNA) (Hestrin and Galarreta 2005). Dendrites of GABAergic interneurons are interconnected by connexin 36 gap junctions and form complex inhibitory neural networks that provide critical modulation of frontal lobe output (Bennett and Zukin 2004; Hestrin and Galarreta 2005). Disrupting GJD2 expression produces a loss of electrical coupling among GABAergic interneurons and leads to ineffective generation of fast synchronized oscillations in neocortical neural networks (Bennett and Zukin 2004). Clinically, the loss of GABAergic control of fast and ultrafast rhythms can produce abnormalities in neuronal processing, sensory perception, motor performance, learning, attention, and memory consolidation in brain neocortex (Bennett and Zukin 2004).

In order to better understand abnormal GABAergic transmission in HIV infected patients several issues need to be elucidated: 1) It is not known whether replicating HIV in the brain drives GABAergic changes, or whether suppressing virus replication with cART normalizes the change; 2) Although HAND without HIVE is the dominant clinicopathological sequence in virally suppressed patients (Gelman et al. 2012a; Gelman 2015), the importance of GABAergic anomalies in these patients and the role of HIVE need to be elucidated; 3 ) It is not clear what type of neuropsychological dysfunction, if any, is associated with GABAergic changes in HAND. 4) It remains unclear whether loss of GABAergic marker protein represents the death of inhibitory neurons (i.e., classical 
pathological neurodegeneration) as is often suggested, or instead reflects modified expression of GABAergic marker proteins in viable interneurons (i.e., accommodation due to synaptic plasticity) (Akbarian et al. 1995; Volk et al. 2000; Gelman et al. 2006). 5) Basic brain regional anatomy and circuit-level dysfunction of GABAergic inhibitory networks need to be better characterized in HIV infected subjects (Gelman et al. 2012a). 6) Because of the role of GABA in regulating cerebral blood flow, it is not known whether GABAergic anomalies contribute to abnormal neurovascular biology in HIV infected patients (Strazza et al. 2011). To address these issues we evaluated neurochemical markers of GABAergic transmission in 449 brain specimens obtained from HIV-infected subjects, many of whom underwent antemortem neuropsychological testing.

\section{Materials and Methods}

\section{Human Brain Specimens}

GABAergic mRNA concentrations were measured in the dorsolateral prefrontal cortex (DLPFC) of 515 human postmortem brain specimens obtained from National NeuroAIDS Tissue consortium (NNTC) (Morgello et al. 2001). This particular patient cohort was described in a previous communication (Gelman et al. 2012b). DLPFC is of prime relevance because the functional output of this brain sector is abnormal in HAND (Woods et al. 2009). 449 of the patients were infected with HIV-1 and 66 were demographically comparable HIV seronegative decedents (Gelman et al. 2006, 2012b) (Table S1). 131 out of 449 of the HIV-infected patients died before 1997 and/or before cART was introduced to the patient. cART status was defined as being active if the patient had taken at least 2 nucleoside/nucleotide reverse transcriptase inhibitors (NRTIs) or 1 nonnucleoside reverse transcriptase inhibitor (NNRTI) and 1 protease inhibitor within 1 year of death (Gelman et al. 2013). 290 out of 313 patients with documented cART status were cART active within 1 year of death. 219 out of 449 of the HIV-infected patients participated in longitudinal clinical studies after 1999 during the cART era, which included neuropsychological testing in the 6 months before death. For the subjects who were studied clinically, written consent was obtained for subjects at four collection sites in the USA. The following offices maintained institutional review boards (IRBs) that provided oversight for the protection of human subjects: 1) The University of Texas Medical Branch Office of Research Subject Protections; 2) Mount Sinai Medical Center Program for the Protection of Human Subjects; 3) University of California, San Diego Human Research Protections Program; 4) University of California, Los Angeles Office of the Human Research Protection Program.

\section{Neuropsychological Testing and Substance use Survey}

Neuropsychological evaluations and substance use surveys were performed every 6 months using the protocol of the National NeuroAIDS Tissue Network (NNTC). The NNTC neurocognitive test battery included assessment of seven cognitive domains: 1) verbal fluency (Controlled Oral Word Association Test, COWAT- FAS); 2) speed of information processing (Wechsler adult intelligence scale, WAIS-III Digit Symbol and Symbol Search subtest and Trail Making Test Part A); 3) learning (Hopkins Verbal Learning Test Revised, HVLT-R; Total Trial 1-3 Recall and Brief Visuospatial Memory Test - Revised, BVMT-R, Total Trial 1-3 Recall); 4) memory (HVLT-R Delayed Recall, BVMT-R Delayed Recall); 5) executive functions (Wisconsin Card Sorting Test, WCST 64-item version; perseverative responses and TMT Part B) (6) attention and work- ing memory (WAISIII Letter-Number Sequencing, PASAT-50); 7) motor (Grooved Pegboard Test, dominant and nondominant hand performances). The Wide Range Achievement Test-3 (WRAT-3) Reading subtest was administered as an estimate of premorbid verbal intellectual functioning (Woods et al. 2004). The Psychiatric Research Interview for Substance and Mental Disorders (PRISM) or the Composite International Diagnostic Interview (CIDI) was used to obtain self-reported lifetime histories of substance abuse and dependence (Robins et al. 1988; Morgello et al. 2001). A diagnosis of HAND was assigned guided by American Academy of Neurology criteria as modified by the Frascati Criteria.

\section{Brain Specimen Dissection and Neuropathological Data}

Brain specimens obtained fresh at autopsy were bisected in the sagittal plane. One hemisphere was sliced fresh and the slices were stored at $-80{ }^{\circ} \mathrm{C}$. The other hemisphere was immersed in a $20 \%$ formalin solution at $4{ }^{\circ} \mathrm{C}$ for 10 days. 200-300 mg of frozen grey matter from the dorsolateral prefrontal cortex in Brodmann area 9 or 8 was dissected for mRNA and protein extraction. Other brain regions were dissected for regional comparisons as indicated in the figures and tables. Frozen samples were kept on dry ice and placed in pre-weighed and pre-cooled vials and were stored at $-80{ }^{\circ} \mathrm{C}$. Specimens were assayed in batches that were freshly thawed. The neuropathological diagnoses were obtained using the brain sampling and staining protocols of the NNTC (Morgello et al. 2001). The diagnosis of HIV encephalitis (HIVE) was made according to established criteria (Budka 1991; Gelman et al. 2013).

\section{Quantitative Real-Time PCR}

About $100 \mathrm{mg}$ of brain tissue was homogenized in $1 \mathrm{ml}$ of QIAzol reagent (RNeasy Mini Kit, Qiagen, Valencia, CA) for 
isolation of total RNA according to the standard manufacturer's protocol. Single strand cDNA was prepared using Bio-Rad iScript cDNA synthesis Kit (Bio-Rad, Hercules, CA). 2 ug of brain mRNA were mixed with 8 ul of $5 x$ iScript reaction mix, 2 ul of iScript reverse transcriptase and total volume was adjusted to $40 \mathrm{ul}$ with nuclease-free water. The reaction mixture was incubated in the Bio-Rad I-cycler programmed for $5 \mathrm{~min}$ at $25{ }^{\circ} \mathrm{C}, 30 \mathrm{~min}$ at $42{ }^{\circ} \mathrm{C}, 5 \mathrm{~min}$ at $85{ }^{\circ} \mathrm{C}$ and held at $4{ }^{\circ} \mathrm{C}$. For quantitation of the mRNA primers and FAM probes were obtained for GAD1 (Cat. No. Hs01065893_m1), GAD2 (Cat. No. Hs00609534_m1), GJD2 (Cat. No. Hs00706940_s1), ISG15 (Cat. No. Hs00192713 m1), MX1 (Cat.No. HS00182073_m1), IRF1 (Cat.No. Hs00971959_m1), GZMB (Cat.No. Hs01554355 m1), CD4 (Cat.No. Hs01058407_m1), CD8A (Cat.No. Hs01555600_m1), CD19 (Cat.No. Hs99999192_m1), CD68 (Cat.No. Hs00154355_m1), CD163 (Cat.No. Hs01016661_m1), VWF (Cat.No. Hs00169795_m1), PECAM1 (Cat.No. Hs00169777_m1), from Applied Biosystems, Foster City, CA, USA), Glyceraldehyde 3-phosphate dehydrogenase (GAPDH, Cat. No. Hs99999905_m1) was used as the normalizing gene. For a $20 \mathrm{ul}$ reaction mixture $1 \mathrm{ul}$ of cDNA was mixed with 10 ul of TaqMan 2x PCR Master Mix (Applied Biosystems), 8 ul $\mathrm{H} 2 \mathrm{O}$ and $1 \mathrm{ul}$ $\mathrm{ABI} 20 \mathrm{x}$ primer (gene of interest or GAPDH). PCR reactions were performed on Mastercycler RealPlex (Eppendorf, Germany) under the following conditions: activation $\left(10 \mathrm{~min}\right.$ at $\left.95{ }^{\circ} \mathrm{C}\right)$, and 40 cycles of denaturation $\left(15 \mathrm{~s}\right.$ at $\left.95{ }^{\circ} \mathrm{C}\right)$ - annealing/extension $\left(60 \mathrm{~s}\right.$ at $\left.60{ }^{\circ} \mathrm{C}\right)$. Experiments were performed on 96-well plates, each sample was run in duplicates, triple negative controls and standard calibrators were run on each plate. Duplicate $C_{T}$ values were analyzed using the comparative $C_{T}\left(\Delta \Delta C_{T}\right)$ method as described by the manufacturer (Applied Biosystems). The amount of targets $\left(2^{-\Delta \Delta C T}\right)$ was obtained by normalizing to endogenous reference (GAPDH).

\section{Immunoblotting}

Frozen tissue samples were homogenized in $3 \mathrm{x}$ volume of buffer $\left(10 \mathrm{mM}\right.$ Tris- $\mathrm{HCl}, 5 \mathrm{mM} \mathrm{MgCl}_{2}, 0.5 \mathrm{mM}$ dithiothreitol (DTT), $0.03 \%$ Triton X-100, pH 7.8) by silica beads beating (2 times for $20 \mathrm{~s}$ ) and sonication (2 times for $20 \mathrm{~s}$ ) with tubes kept on ice for $5 \mathrm{~min}$ between sessions. Protein concentration was determined using Bio-Rad Protein Assay (Bio-Rad Laboratories, Hercules, CA) and bovine serum albumin (BSA) standards. 15 ug of total protein were loaded into Criterion Precast 18-well 4-20\% gradient Tris-HCl gel (Bio-Rad Laboratories, Hercules, CA) for SDS-PAGE electrophoresis and run at 180 volts for $45 \mathrm{~min}$. Separated proteins were transferred to PVDF membrane (Amersham
Biosciences, Piscataway, NJ) in $10 \mathrm{mM}$ Tris-glycine buffer containing $10 \%$ methanol at $75 \mathrm{~W}$ for $1 \mathrm{~h}$ at $4{ }^{\circ} \mathrm{C}$. The membrane was blocked in $5 \%$ non-fat milk in Tris buffer Saline (TBS-T; $50 \mathrm{mM}$ Tris- $\mathrm{HCl}$, $150 \mathrm{mM} \mathrm{NaCl}, 0.01 \%$ Tween-20) for $1 \mathrm{~h}$ and incubated with mouse anti-GAD67 (Cat. No. ab22050, Abcam) or mouse anti-GAPDH (Cat.No. sc-47724, Santa Cruz Biotechnologies, Santa Cruz, CA, USA) primary antibodies diluted in blocking solution overnight at $4{ }^{\circ} \mathrm{C}$. The membrane was washed three times in TBS-T and incubated with sheep anti-mouse IgG antibodies (Cat. No. Na931V, GE Healthcare, UK) diluted 1:1000 in blocking solution for $2 \mathrm{~h}$. Then the membrane was incubated with Enhanced Chemiluminescence Detection Reagent (Amersham Biosciences, Piscataway, NJ) for 2 min and exposed to Kodak BioMax XAR film (Kodak, Rochester, NY). Film was developed and scanned and bands densities were measured using ImageJ software (NIH, Bethesda, MD, USA).

\section{Immunohistochemistry}

After formalin fixation, blocks of brain tissue were trimmed and embedded in paraffin wax. Sections of tissue (6-8 um) were mounted on glass slides (Superfrost Plus Gold, Erie Scientific, Portsmouth, NJ). The sections were deparaffinized in xylene, rehydrated in graded alcohols and immersed in $0.01 \mathrm{M}$ sodium citrate buffer heated in a microwave oven for $20 \mathrm{~min}$. Unspecific binding was blocked for 20 min with $1 \%$ normal serum and then the sections were incubated overnight at $4{ }^{\circ} \mathrm{C}$ with primary antibodies diluted in blocking solution. Following primary antibodies were used: anti-GAD67 (Cat. No. ab26116, Abcam, Cambridge Science Park, Cambridge, England) diluted 1:1000, anti-CD31 (Cat. No. M0823, Dako, Via Real, Carpinteria, CA, USA) diluted 1:30, anti-granzyme B (Cat.No. GB-7, Sanquin, Sanquin Blood Supply, Amsterdam, Netherlands) diluted 1:50, anti-CD8A (Cat.No. M710301-2, Dako) diluted 1:50, anti-CD68 (Cat.No. M0814, Dako) diluted 1:50. Next day tissue was treated with $3 \%$ hydrogen peroxide for $10 \mathrm{~min}$ and rinsed with TBS-T $(50 \mathrm{mM}$ Tris- $\mathrm{HCl}, 150 \mathrm{mM} \mathrm{NaCl}$, $0.05 \%$ Tween-20). Appropriate anti-mouse or anti-rabbit Vectastain secondary antibodies were applied for $1 \mathrm{~h}$, followed by Vectastain $\mathrm{ABC}$ and Vectastain $\mathrm{DAB}$ color development using peroxidase-diaminobenzidine reactions (Vector Laboratories, Burlingame, CA). Sections were dehydrated and mounted using Permount (Fisher Scientific, Hampton, NH). Composite microscopic fields were acquired by combining contiguous images taken using an Olympus DP71 camera and Olympus DP controller software (Olympus America Inc, Center Valley, PA, USA). 


\section{Immunofluorescence}

Sections for immunofluorescence underwent deparaffinization and antigen retrieval treatment as described above. After antigen retrieval, tissue was permeabilized for 15 min with $0.1 \%$ Triton-X in Phosphate buffer saline (PBS) and treated with ImageT FX signal enhancer (Invitrogen Molecular Probes, Eugene, Oregon) for $30 \mathrm{~min}$. Unspecific binding was blocked for $1 \mathrm{~h}$ with $1 \%$ normal serum and then the sections were incubated overnight at $4{ }^{\circ} \mathrm{C}$ with primary antibodies diluted in blocking solution. Primary antibodies were used: mouse anti-GAD67 (Cat. No. mab5406, EMD Millipore Corporation, Billerica, MA, USA) diluted 1:100, rabbit antiparvalbumin (Cat. No. ab11427, Abcam) diluted 1:1000, mouse anti-dopamine receptor 2 (Cat.No. sc-5303, Santa Cruz Biotechnologies, Santa Cruz, CA, USA) diluted 1:50. Next day sections were rinsed in with TBS-T (50 mM Tris$\mathrm{HCl}, 150 \mathrm{mM} \mathrm{NaCl}, 0.05 \%$ Tween-20) and mixture of secondary antibodies diluted 1:500 in PBS were applied for $3 \mathrm{hs}$ (AlexaFluor 488 goat anti-mouse (Cat.No. A11029), and AlexaFluor 594 goat anti-rabbit (Cat.No.A11037), Life Technologies, Grand Island, NY, USA). After incubation with secondary antibodies, sections were rinsed with TBS-T and treated with Sudan Black B (0.5 in $70 \%$ ethanol) for 20 min. Then sections were rinsed in $\mathrm{ddH} 2 \mathrm{O}$, air dried and mounted using ProLong Gold with DAPI mounting media (Life Technologies). Confocal images were then acquired with a Zeiss LSM-510 Meta confocal microscope with $63 \mathrm{x} / 1.40$ Oil DIC (WD=0.19 mm) objective (Carl Zeiss Microscopy, Peabody, MA, USA) and processed using LSM Image Browser (Zeiss).

\section{Statistical Analysis}

Statistical analysis was performed using Microsoft Excel 2010 (Microsoft Corporation, Redmond, Washington, DC, USA), and GraphPad 6 (GraphPad Software, Inc., La Jolla, CA, USA). Two-tailed Student's $t$-test was used to compare two groups. One-way analysis of variance with post hoc Scheffé's or uncorrected Fisher's LSD tests were used to compare the mean of one group to the mean of other groups. Pearson's correlation was used to analyze correlations between GABAergic mRNAs level and neurovirological, neuroimmunological and neurocognitive measurements. These data were $\log _{10}$ transformed to normalize the distributions. Significance of correlation was corrected for the false discovery rate using the Bonferroni correction. Significance was set at $\alpha=0.05$. Fisher z-transformation was used to estimate the significance of the difference between two correlation coefficients found in two independent samples. Multiple regression models were used to determine whether potentially confounding factors such as age, gender, race, and drugs of abuse affected GABAergic mRNA.

\section{Results}

\section{GAD1, GAD2 and GJD2 mRNA Expression}

Three markers of GABAergic interneurons in the DLPFC were abnormally expressed in the HIV-infected patients compared to normal uninfected controls. mRNAs that correspond to GAD67, GAD65, and connexin 36 proteins all were significantly lower in the HIV positive subjects versus the HIV negative controls (for GAD1, $-26 \%$ and $p<10^{-4}$, for $G A D 2,-22 \%$ and $p<0.001$, for GJD2, $21 \%$ and $p=0.0043$; Fig. 1a-c). When HIV-infected subjects were divided into those that died before the era of cART versus after, all three mRNAs were significantly lower in both eras (by 30 and $24 \%$ for GAD1, 19 and $24 \%$ for $G A D 2,17$ and $22 \%$ for GJD2; Fig. 1d-f). Between group analysis using one-way analysis of variance (ANOVA) and post hoc Scheffé's test yielded $\mathrm{F}=13.27$ and $p<10^{-4}$ for GAD $1, \mathrm{~F}=5.957$ and $p=0.0028$ for $G A D 2$, and $\mathrm{F}=4.425$ and $p=0.012$ for GJD2. The pre- and post-cART era groups did not differ significantly from each other. When HIV-positive subjects were sorted according to whether or not they had HIV encephalitis (HIVE) at autopsy, the mRNAs were significantly lower than HIV-negatives in the patients without and with HIVE both (by 26 and $23 \%$ for GAD1, 21 and $27 \%$ for $G A D 2,20$ and $24 \%$ for GJD2; Fig.1g-i). Between group analysis using one-way analysis of variance (ANOVA) and post hoc Scheffé's test yielded $\mathrm{F}=12.55$ and $p<10^{-4}$ for GAD1, $\mathrm{F}=5.960$ and $p=0.0028$ for $G A D 2$ and $\mathrm{F}=4.313$ and $p=0.014$ for GJD2. The groups with and without HIVE did not differ from each other significantly with respect to these mRNAs.

\section{Regional Distribution of the GABAergic Anomaly in the CNS}

To determine if specific brain regions were selectively vulnerable to having low GABAergic markers, we measured GAD1 mRNA in a total of 16 sectors of the human CNS, including 7 neocortical regions, 6 subcortical regions, cerebellum and spinal cord (Fig. 2). Six HIV negative controls and 6 HIV-infected subjects were selected for study based upon their GABAergic mRNA values in the DLPFC. There was substantial variation in GAD1 mRNA expression between brain regions (F (16, $218)=7.17, p<10^{-4}$, two-way ANOVA). In almost all regions GADI mRNA was lower in the HIV infected subjects, including neocortex, neostriatum and cerebellar lobule. Exceptions were spinal cord, paleostriatum (globus pallidus) and hippocampus in which the lower $G A D 1$ expression was not statistically significant. 

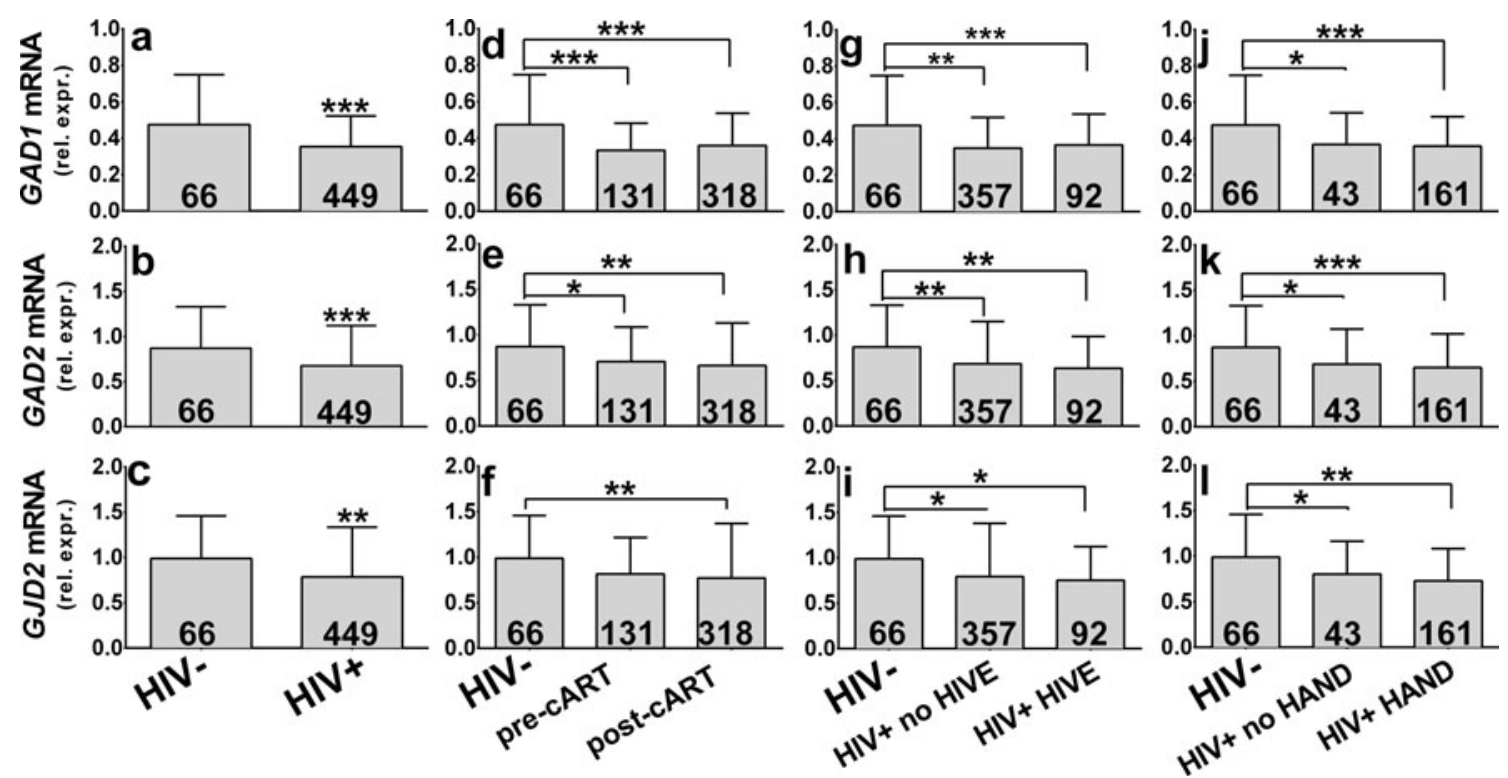

Fig. 1 Glutamate decarboxylase $1(G A D 1)$, glutamate decarboxylase 2 (GAD2), and connexin 36 (GJD2) mRNAs in the dorsolateral prefrontal cortex of 449 HIV infected and 66 HIV seronegative patients (Panels a 1). "Rel. exp." on the ordinates denotes mRNA expression relative to GAPDH mRNA. The number of subjects in each group is given in the bars. Panels a - c. GABAergic mRNA expression was significantly lower in the HIV infected patients. Panels $\mathrm{d}-\mathrm{f}$. HIV-positive subjects were divided into subjects who died before and after the introduction of cART. mRNA expression was significantly lower in pre-cART and post-cART HIV patients as compared to the uninfected comparison group. There were no significant differences between pre-cART and
post-cART groups. Panels g - i. HIV-positive subjects were divided according to neuropathological diagnosis of HIV-encephalitis (HIVE). All three mRNAs were significantly lower in patients without HIVE and with HIVE when compared to the uninfected comparison group. There were no significant differences between HIVE and no HIVE groups. Panels j 1. HIV-positive subjects were divided according to the diagnosis of HIVassociated neurocognitive disorders (HAND). Compared to the uninfected comparison group, all three mRNAs were significantly lower in patients without HAND and with HAND. There were no significant differences between HAND and no HAND groups. Mean \pm standard deviation. ${ }^{*} p<0.05 ; * * p<0.01 ; * * * p<0.001$

\section{Relationship of GABAergic mRNAs to Demographic Factors and Substance use History}

Some studies have suggested that substance abuse can produce changes in GABAergic transmission (Zhang et al. 2006; Satta et al. 2008). When sorted according to self-reported history of drug abuse, HIV-infected subjects with and without drug abuse history contained significantly lower values for all three GABAergic markers mRNAs compared to controls (by 25 and $28 \%$ for GAD1, 24 and $27 \%$ for GAD2, 25 and $27 \%$ for GJD2; Fig. S1 a-c) . Between group analysis using one-way ANOVA yielded $\mathrm{F}=9.242$ and $p<10^{-4}$ for GAD1, $\mathrm{F}=7.124$ and $p=0.001$ for $G A D 2$, and $\mathrm{F}=8.12$ and $p=0.0004$ for GJD2. Between-group comparisons revealed no difference between subjects with and without a history of drug abuse.

Several CNS conditions can influence the expression of the above transcripts. To examine whether such confounders impact the concentration of GABAergic mRNAs, HIVpositive subjects were sorted according to whether or not they had comorbid CNS disease (such as progressive multifocal leukoencephalopathy (PML), primary CNS Lymphoma (PCNSL), CMV ventriculoencephalitis (CMVE), toxoplasmosis Encephalitis (TE), meningitis (such as aseptic leptomeningitis, bacterial leptomeningitis, cryptococcal meningitis), or hemorrhages). All three mRNAs were significantly lower in both groups (without and with comorbidities) of HIV-positives (by 25 and $28 \%$ for GADI, 22 and $29 \%$ for GAD2, 23 and $22 \%$ for GJD2; Fig. S3 g - i). Between group analysis using one-way analysis of variance (ANOVA) and post hoc Tukey's test yielded $\mathrm{F}=12.34$ and $p<10^{-4}$ for $G A D 1, \mathrm{~F}=8.418$ and $p=0.0003$ for $G A D 2$ and $\mathrm{F}=9.648$ and $p<10^{-4}$ for GJD2. The groups without and with comorbid CNS diseases tissue did not differ from each other significantly with respect to these mRNAs.

Age, gender and ethnicity are other potential variables that could affect GABAergic genes expression in human brain specimens (Pinto et al. 2010; Seney et al. 2013). Multiple regression models showed that potentially confounding co-variables including drug abuse, age, gender, and race were not significantly related to GAD1 and GJD2 mRNAs (not shown). For GAD2 mRNA statistical models suggested a significantly higher level in males $(p=0.0027)$, but overall results for GAD2 were not changed when the results were corrected statistically for the influence of gender (data not shown). 


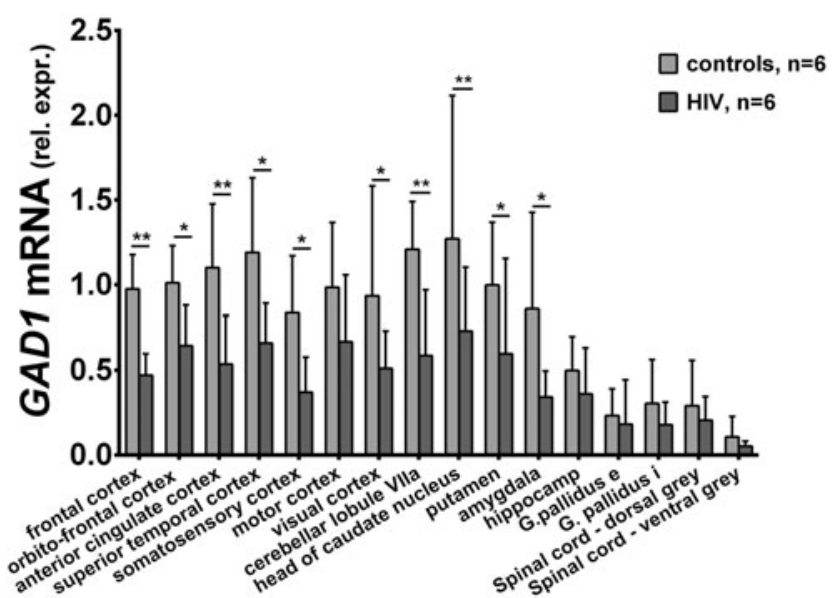

Fig. 2 Glutamate decarboxylase 1 mRNA (GADI) expression was measured in 16 different brain regions obtained from 6 HIV-infected patients and 6 uninfected patients. Using two-way ANOVA, only variation in GAD1 mRNA concentrations between brain regions was significant $\left(\mathrm{F}(16,218)=7.17, p<10^{-4}\right)$. GADl was lower in the HIV infected brain specimens in most regions including neocortex, cerebellum and neostriatum (caudate nucleus and putamen). Uncorrected Fisher's LSD tests were significant for dorsolateral prefrontal $(-51.7 \%, p=0.010)$, anterior cingulate $(-51.2 \%, p=0.009)$, superior temporal $(-44.5 \%$, $p=0.013)$, somatosensory $(-55.6 \%, p=0.042)$, and visual cortices ($45.4 \%, p=0.038)$, cerebellar lobule VIIa $(-51.5 \%, p=0.003)$, head of caudate nucleus $(-42.5 \%, p=0.009)$, putamen $(-40.1 \%, p=0.049)$, and amygdala $(-60 \%, p=0.012)$. Mean decrease of GAD1 mRNA expression in the orbitofrontal cortex was $-36.2 \%$ and almost reached significance $(p=0.0728)$. Spinal cord, paleostriatum, and hippocampus also had slightly lower GAD1 in the HIV infected brain specimens, but those differences were not significant statistically. Relative expression of $G A D 1$ mRNA was normalized to GAPDH mRNA. Mean \pm standard deviation. $*^{*}<0.05 ; * * p<0.01 ; * * * p<0.001$

\section{Relationship Between GABAergic mRNAs, Clinical Virology and Neurovirology}

Correlation coefficients between brain HIV RNA loads and GABAergic mRNAs in the DLPFC were not significant (Table S2). When GABAergic mRNAs were correlated with HIV RNA in blood plasma, the correlation coefficient was negative and statistically significant for GAD2 mRNA $(n=264, \mathrm{r}=-0.1372, p=0.0258)$, but not the other mRNAs. When GABAergic mRNAs were correlated with HIV RNA in the CSF samples that were available, correlation coefficients were negative and significant for two GABAergic mRNAs (GAD2: $n=191, \mathrm{r}=-0.1582, p=0.0286$; GJD2: $n=191, \mathrm{r}=-$ $0.1801, p=0.0127)$. Neurovirological correlations in patients with HIVE often differ from those without HIVE (Gelman et al. 2012a, 2013). To determine whether HIVE influenced the neurovirological correlation with GABAergic mRNAs, patients with and without HIVE were evaluated separately. GAD1 mRNA was negatively and significantly correlated with brain HIV RNA in the subjects without HIVE, whereas the correlation for patients with HIVE had a positive coefficient that almost reached significance (Table S2). Fisher $r$ to $\mathrm{Z}$ transformation showed that the two oppositely sloped regression lines were statistically different, comparing patients with HIVE versus without it (two-tailed $p=0.0278$ ). These results suggest that HIVE, which produces high loads of replicating HIV in the brain and increased inflammation, tends to blunt the decrease of the GABAergic mRNAs in HIV infected patients.

When HIV-infected subjects were divided into those that have low versus high viral load in the cerebrospinal fluid (CSF), all three mRNAs were significantly lower in both groups of HIV-positives (by 22 and $36 \%$ for GAD1, 19 and $36 \%$ for GAD2, 22 and $36 \%$ for GJD2; Fig. S3 a - c). Between group analysis using one-way analysis of variance (ANOVA) and post hoc Tukey's test yielded $\mathrm{F}=12.44$ and $p<10^{-4}$ for $G A D 1, \mathrm{~F}=10.33$ and $p<10^{-4}$ for $G A D 2$, and $\mathrm{F}=14.37$ and $p<10^{-4}$ for GJD2. The low and high viral load in the CSF groups did not differ significantly from each other. When HIV-positive subjects were sorted according to low versus high viral load in the frontocortical grey matter, all three mRNAs were significantly lower in both groups of HIV-positives (by 27 and $25 \%$ for GAD1, 20 and $23 \%$ for GAD2, 22 and $24 \%$ for GJD2; Fig. S3 d - f). Between group analysis using one-way analysis of variance (ANOVA) and post hoc Tukey's test yielded $\mathrm{F}=12.29$ and $p<10^{-4}$ for $G A D 1, \mathrm{~F}=5.673$ and $p=0.0037$ for $G A D 2$ and $\mathrm{F}=9.642$ and $p<10^{-4}$ for GJD2. The groups with low versus high viral load in brain tissue did not differ from each other significantly with respect to these mRNAs.

\section{Relationship of GABAergic mRNAs to Neurocognitive Impairment}

Abnormal GABAergic transmission occurs in diseases that can adversely affect neurocognitive test performance, especially on tasks that are driven by the DLPFC (Volk et al. 2000; Woods et al. 2009). To address whether the GABA disturbance was related to HIV-associated neurocognitive disorders (HAND), we sorted the subjects according to the diagnosis of HAND and the GABAergic mRNAs were compared in the two groups. All three GABAergic mRNAs were significantly lower in the subjects with HAND relative to the uninfected controls; the same result was obtained in the subjects without HAND (Fig. 1j-1). Between group analysis using oneway analysis of variance (ANOVA) and Scheffe's post hoc test yielded $\mathrm{F}=8.420$ and $p=0.0003$ for $G A D 1, \mathrm{~F}=7.384$ and $p<0.0008$ for $G A D 2$, and $\mathrm{F}=10.51$ and $p<10^{-4}$ for GJD2. There were no significant differences between HAND and no HAND groups. When subjects with HAND were sorted according to the severity of impairment, all three mRNAs were significantly lower in patients diagnosed with HIV-associated dementia (HAD) and Mild Neurocognitive Disorder (MND) compared to seronegative controls (by 24 and $18 \%$ for GAD1, 32 and $22 \%$ for GAD2, 29 and $22 \%$ 
for GJD2; Fig. S1 d-f). One-way analysis of variance (ANOVA) yielded $\mathrm{F}=5.294$ and $p=0.0015$ for $G A D 1$, $\mathrm{F}=6.688$ and $p=0.0002$ for $G A D 2$, and $\mathrm{F}=7.215$ and $p=0.0001$ for GJD2. Groups of HIV-positives with different degrees of cognitive impairment did not differ from each other. There were no significant differences between HIVpatients diagnosed with Asymptomatic Neurocognitive Impairment (ANI) and controls.

Using the composite neurocognitive impairment $\mathrm{T}$ score, Pearson's correlation analysis showed no significant correlation with GABAergic mRNAs in the DLPFC. When GABAergic mRNAs were compared to performance in distinct functional domains that were assessed in the test battery, there was a significant correlation between low GABAergic mRNAs and worse performance on the verbal fluency task $(p=0.0036$ for GAD1 mRNA, $p=0.0005$ for GAD2 mRNA, $p=0.0013$ for GJD2 mRNA) after correction for multiple comparisons (Table 1).

\section{Relationships Between GABAergic mRNAs, Endothelial and Immune Cell Markers}

In addition to the potential influence of CNS virus replication, it has been suggested that neuroimmune anomalies including changes in the neurovascular unit (Strazza et al. 2011) and heightened interferon responses could drive HAND (Gelman et al. 2012a). When mRNAs that mark neurovascular and neuroimmune type changes were examined, strong correlations were found between low GABAergic mRNAs and high expression of the endothelial cell markers PECAMI and $V W F$ (Table 2). Low GABAergic mRNAs were correlated significantly, but less strongly with high expression of neuroimmune type markers including a prototypical type 1 interferon response gene (MX1), a type 2 interferon response gene (IRF1), macrophages (CD163 and CD68), CD8+ cytotoxic $\mathrm{T}$ lymphocytes (CD8A), and natural killer cells (GZMB). In contrast, markers for B lymphocytes (CD19) and CD4+ T lymphocytes (CD4) were not significantly correlated with low GABAergic mRNAs. Immunohistochemical staining of brain tissue for marker antigens corresponding to endothelial cells, macrophages, CD8+ lymphocytes, and natural killer cells generally confirmed these associations at protein and cellular levels (Fig. 3).

\section{Interrelationships Between GABAergic, Dopaminergic and Enkephalinergic mRNAs}

Dopaminergic and enkephalinergic neural transmission both have been shown to be neurochemically abnormal in the DLPFC of this HIV infected autopsy cohort (Gelman et al. 2012b). These neurotransmitter type changes were compared to the results for GABAergic mRNAs (Fig. S2). In seronegative controls GAD1 mRNA was positively and significantly correlated with the transcript of a key dopaminergic receptor $(D R D 2 L)$ that was previously shown to be regulated in the DLPFC of HIV infected patients $(n=66, \mathrm{r}=0.267, p=0.03)$. In contrast, the same correlation analysis in the HIV infected subjects produced a significant negative correlation coefficient $(n=449, \mathrm{r}=-0.157$, $p=0.0008$ ). Using bootstrapping and Fisher $\mathrm{z}$-transformations these correlations were significantly different from each other (two-tailed $p=0.011$ ). Thus, reduced expression of GABAergic markers is significantly related to higher $D R D 2 L$ expression in the HIV infected subjects but not in seronegative controls. When HIV-positive subjects were grouped according to the clinical diagnosis of HAND versus no HAND there was a significant correlation between GABAergic mRNAs and DRD2L in the subjects with HAND $(n=197, \mathrm{r}=-0.183, p=0.01)$. Infected subjects without HAND also exhibited a negatively sloped regression line, but the correlation was not significant statistically ( $n=43, \mathrm{r}=-0.159, p=0.306)$, probably due to the more limited number of available subjects in that subgroup.

Table 1 GABAergic transcripts correlated with normalized neurocognitive T-scores

\begin{tabular}{|c|c|c|c|c|c|c|c|}
\hline \multicolumn{2}{|l|}{$H I V+$} & \multicolumn{2}{|c|}{$G A D 1$ mRNA } & \multicolumn{2}{|c|}{$G A D 2$ mRNA } & \multicolumn{2}{|c|}{ GJD2 mRNA } \\
\hline Neurocognitive test domain & $n$ & $r$ & $p$ & $r$ & $p$ & $r$ & $p$ \\
\hline Memory & 218 & 0.0322 & 0.6363 & 0.0256 & 0.7070 & 0.0528 & 0.438 \\
\hline Attention and working memory & 212 & 0.0229 & 0.7403 & 0.0791 & 0.2515 & 0.0493 & 0.475 \\
\hline Learning & 219 & 0.0073 & 0.9145 & 0.0653 & 0.3361 & 0.0539 & 0.4274 \\
\hline Motor & 202 & 0.0353 & 0.6180 & 0.0306 & 0.6650 & 0.1124 & 0.1112 \\
\hline Verbal fluency & 216 & 0.1974 & $0.0036^{*}$ & 0.2358 & $0.0005^{*}$ & 0.2171 & $0.0013 *$ \\
\hline Abstract executive & 209 & 0.0614 & 0.3771 & 0.0974 & 0.1606 & 0.0918 & 0.1861 \\
\hline Speed of information processing & 217 & 0.0377 & 0.5810 & 0.0375 & 0.5827 & 0.0461 & 0.4993 \\
\hline Global T-Score & 196 & 0.0793 & 0.2690 & 0.1003 & 0.1619 & 0.1072 & 0.135 \\
\hline
\end{tabular}

*Asterisk denotes statistically significant $p$ value. GABAergic transcripts were measured in the frontal neocortex

HIV human immunodeficiency virus type 1, HIVE HIV encephalitis, $r$ correlation coefficient 
Table 2 GABAergic transcripts correlated with neuroimmunological and endothelial markers

\begin{tabular}{|c|c|c|c|c|c|c|}
\hline \multirow[t]{2}{*}{$\mathrm{HIV}+(n=449)$} & \multicolumn{2}{|c|}{ GAD1 mRNA } & \multicolumn{2}{|c|}{ GAD2 mRNA } & \multicolumn{2}{|c|}{ GJD2 mRNA } \\
\hline & $r$ & $p$ & $r$ & $p$ & $r$ & $p$ \\
\hline$M X 1 \mathrm{mRNA}$ & -0.1375 & $0.0035^{*}$ & -0.1721 & $0.0002 *$ & -0.0465 & 0.3256 \\
\hline ISG15 mRNA & -0.0306 & 0.5178 & -0.0510 & 0.2809 & -0.0186 & 0.6943 \\
\hline IRF1 mRNA & -0.1574 & $0.0008^{*}$ & -0.1504 & $0.0014^{*}$ & -0.0804 & 0.0888 \\
\hline CD68 mRNA & -0.1946 & $0.0000^{*}$ & -0.1732 & $0.0002 *$ & -0.1181 & $0.0123^{*}$ \\
\hline CD163 mRNA & -0.1950 & $0.0000^{*}$ & -0.1081 & $0.0221 *$ & -0.0750 & 0.0563 \\
\hline$C D 8 A$ mRNA & -0.1197 & $0.0112^{*}$ & -0.1821 & $0.0001 *$ & -0.0369 & 0.4354 \\
\hline CD4 mRNA & -0.0808 & 0.3426 & -0.0647 & 0.4476 & 0.0854 & 0.3157 \\
\hline CD19 mRNA & -0.0501 & 0.2895 & -0.0661 & 0.1620 & -0.0173 & 0.7145 \\
\hline$G Z M B$ mRNA & -0.1450 & $0.0021 *$ & -0.1553 & $0.0010^{*}$ & -0.1018 & $0.0310^{*}$ \\
\hline$V W F$ mRNA & -0.2769 & $0.0000^{*}$ & -0.2902 & $0.0000 *$ & -0.0501 & 0.2895 \\
\hline PECAM1 mRNA & -0.3419 & $0.0000^{*}$ & -0.3529 & $0.0000^{*}$ & -0.1980 & $0.0000^{*}$ \\
\hline PENK $m R N A$ & 0.2968 & $0.0000 *$ & 0.3534 & $0.0000^{*}$ & 0.2018 & $0.0000 *$ \\
\hline
\end{tabular}

*Asterisk denotes statistically significant $p$ value. GABAergic, neuroimmunological and endothelial transcripts were measured in the frontal neocortex HIV human immunodeficiency virus type $1, r$ correlation coefficient

Preproenkephalin mRNA (PENK) was shown previously to be lower in HIV infected subjects in this patient cohort (Gelman et al. 2012b). Lower PENK was correlated significantly with low GABAergic expression in HIV infected patients (Table 2).

\section{GAD67 Protein Expression and Tissue Localization}

Western blotting for GAD67 (the protein product of GAD1 transcript) showed that GAD67 expression was lower in 36 representative HIV infected subjects as compared to 12 uninfected subjects (Fig. 4). GAD67-immunostainining in the
DLPFC further illustrated that protein staining intensity was lower in the neocortex of infected subjects (Fig. 5). Stain intensity in DLPFC was lowered diffusely in cell bodies and neural processes of the cortical laminae. Dual staining was performed to determine whether GAD67 and higher DRD2 protein expression were co-localized in the same neurons. Weak GAD67 staining hindered the effort to localize the two antigens in the infected subjects (not illustrated). When we stained for GABAergic cell markers that were more abundantly expressed, inhibitory interneurons in DLPFC often had strong expression of DRD2 in the HIV infected, but not in the seronegative control specimens (Fig. 6b). Dual staining

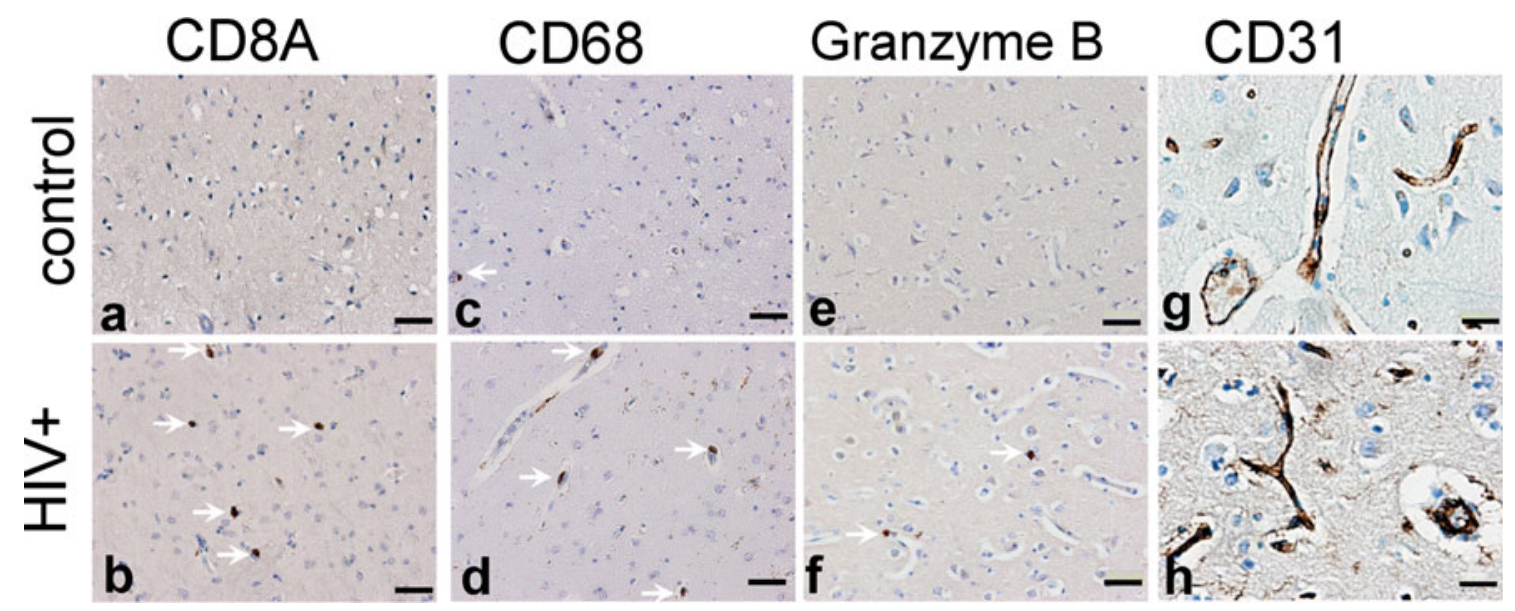

Fig. 3 Neuroimmune and endothelial cells were immunostained in the dorsolateral prefrontal cortex of the HIV-seronegative control (panels a, c, e, g) and HIV-infected subjects $(\mathbf{b}, \mathbf{d}, \mathbf{f}, \mathbf{h}) . \mathrm{CD} 8+$ cytotoxic T cells (arrows, a vs b) and CD68+ macrophages (arrows, $\mathbf{c}$ vs d) were more numerous in specimens from HIV-infected subjects with low GAD1 expression, as suggested by concentrations of the corresponding mRNAs (see Table 2). Granzyme B-containing inflammatory cells were more numerous in specimens from HIV- infected subjects with low GADI expression (arrows, e vs f). Staining for the endothelial cell marker CD31 produced more intense marking of blood vessel walls (arrows) in HIV- infected subjects with low GADl (g vs h). Bars represent 50 um in a $-\mathbf{f}$, and 20 um in $\mathbf{g}$ and $\mathbf{h}$ 


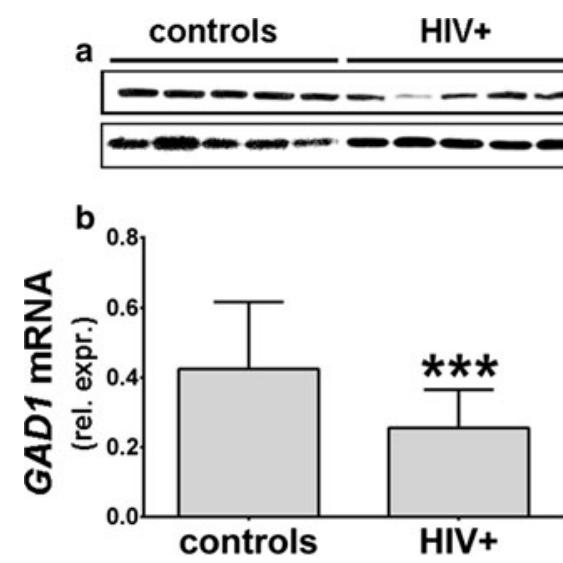

Fig. 4 Glutamate decarboxylase 67 (GAD67) protein and mRNA expression in the dorsolateral prefrontal cortex. GAD67 immunoblotting was done on 36 HIV-infected subjects and 12 seronegative controls. GAPDH blots were done for loading controls. Equal amounts of protein were loaded to each well. GAD67 band intensities were significantly lower in $36 \mathrm{HIV}+$ subjects compared to $12 \mathrm{HIV}$ - subjects $(-51.2 \%, p=0.0001$, panel c). Band

for combinations of two different markers of inhibitory neuronal subpopulations confirmed that the loss of neocortical GAD67 staining occurred in viable interneurons. Examples are illustrated in Fig. 6a, which shows the lack of staining for GAD67 in numerous PV-stained inhibitory neurons of the HIV infected patients. In contrast, the seronegative controls had primarily PV-stained neurons that were positively stained for GAD67. The lack of GAD67 staining in the HIV infected patients was observed almost exclusively in interneurons with morphologically typical-appearing nuclear and perikaryal features.

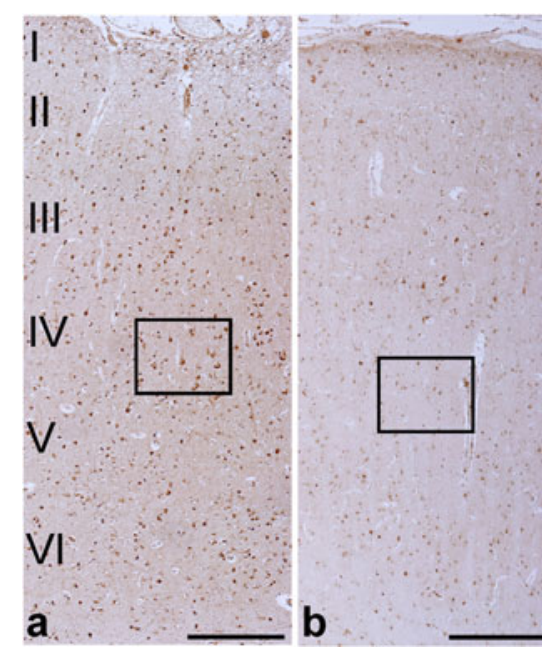

Fig. 5 Glutamate decarboxylase 67 (GAD67) immunoreactivity in brain tissue from an HIV infected patient $(\mathbf{b}, \mathbf{d}, \mathbf{f}, \mathbf{h})$ and a seronegative control (a, c, e, g). Panels a and b illustrate dorsolateral prefrontal cortex (DLPFC) with neocortical laminae labeled I through VI at left. The specimen from the HIV infected subject (b) contains less intense staining in all of the laminae compared to the seronegative patient (a). Boxed areas in a and $\mathrm{b}$ are magnified in $\mathrm{c}$ and $\mathrm{d}$ and illustrate less intense immunostaining

\section{GAD67}

GAPDH

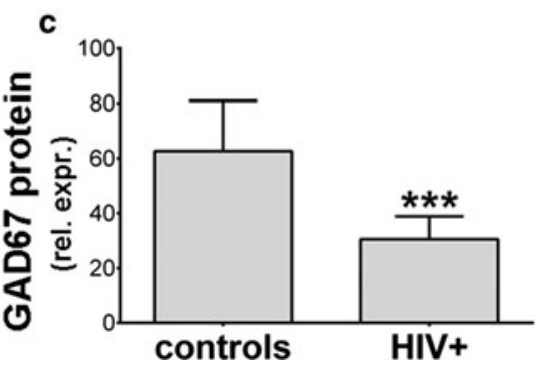

intensities for ten representative subjects are illustrated in panel a. GADI mRNA expression is shown for comparison (b), mean GAD1 mRNA decrease is $-39,8 \%,(p=0.001)$. GAD67 is expressed as relative to the GAPDH band intensity. GAD1 is expressed as relative to GAPDH mRNA. Mean \pm standard deviation is shown. $\mathrm{P}$ values were obtained using the Student's $t$ test. ${ }^{*} p<0.05 ; * * p<0.01 ; * * *<0.001$

\section{Discussion}

This study elucidates the neurochemical, neuropathological, neurocognitive and neurovirological aspects of abnormally low expression of GABAergic markers in HIV-infected patients. Approaching these questions using tissues from a large number of patients imparted strong statistical power. Results with three separate GABAergic mRNAs were consistent, which provided added rigor to the experimental design. Our emphasis on the DLPFC was appropriate given that HAND and other diseases with abnormal GABAergic transmission

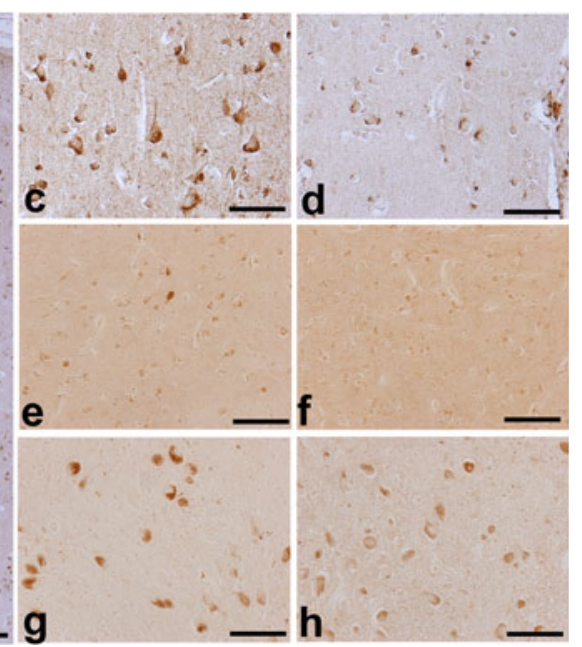

in pyramidal cell cytoplasm, in pear-shaped interneurons and in neuritic processes. In the neostriatum (caudate nucleus), decreased GAD67 immunostaining in the HIV infected subject was not as clear-cut but still was present (e vs f). The neurons in the globus pallidus also were stained less intensely in the HIV infected subject. Note that the number of stained neurons in the HIV infected subject is similar to the seronegative control (g vs h). Scale bars are $200 \mathrm{um}$ in $\mathbf{a}$ and $\mathbf{b}$, and $100 \mathrm{um}$ in $\mathbf{c}-\mathbf{f}$ 

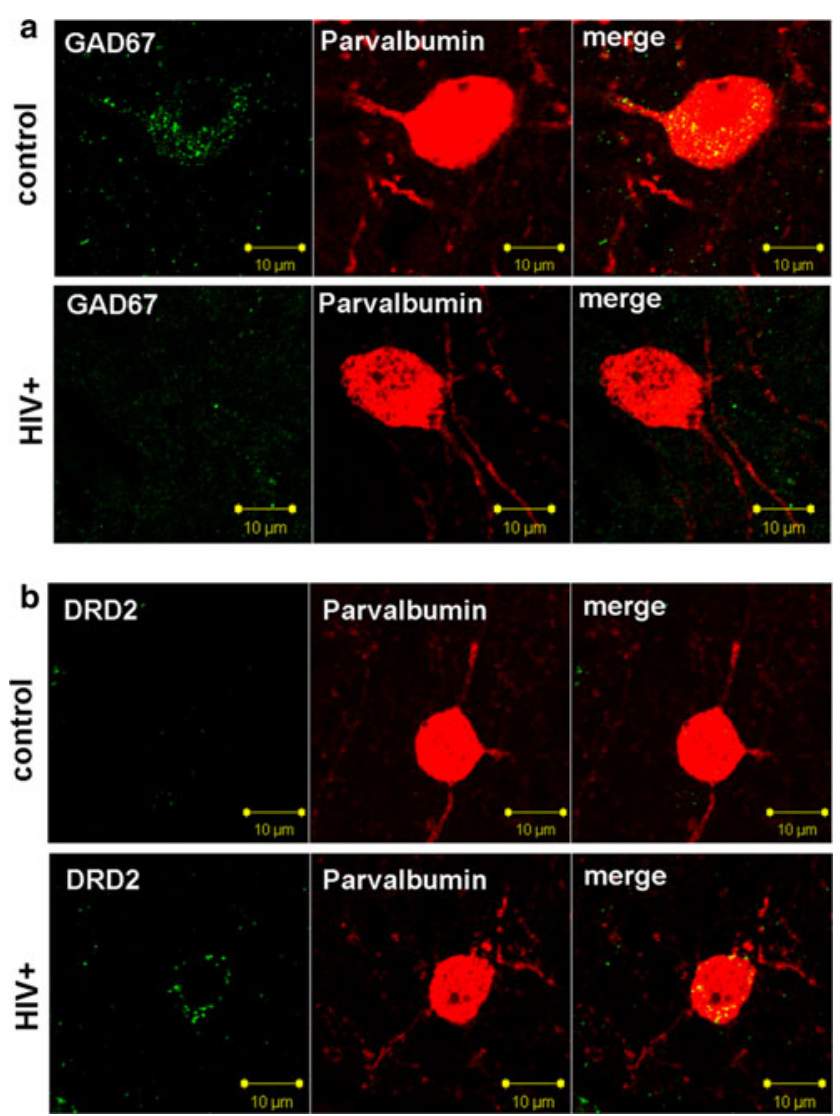

Fig. 6 The six panels in a illustrate that loss of GAD67 staining in HIV infected patients does not correspond to the dropping out of interneurons. Immunofluorescence for interneuronal markers GAD67 and parvalbumin (PV) show that the cortical interneuron from the HIV-infected subject contains less intense GAD67 staining compared to the seronegative control. PV immunoreactivity of interneurons and neuritic processes is of equivalent intensity in these two specimens. The six panels in $\mathbf{b}$ illustrate that HIV-infected patients with HAND had high expression of dopamine receptor 2 (DRD2) in cortical interneurons, when interneuron from a seronegative patient lacks DRD2 staining. PV immunoreactivity of interneurons and neuritic processes is of equivalent intensity in these two specimens. Scale bars are $10 \mathrm{um}$

have neuropsychological deficits that can be traced to abnormal output of the DLPFC (Iudicello et al. 2007; Woods et al. 2009). GABAergic neurotransmission is abnormal in many HIV-infected patients, as previously suggested (Masliah et al. 1995; Fox et al. 1997; Gelman et al. 2012a). Our data on brain virus replication show that the GABAergic anomaly is not correlated with the amount of replicating HIV-1 in the CNS. Further, the data show no significant association with HIVE, which is a neuropathology driven by replicating HIV-1 in the CNS. Also, the anomaly was not normalized in patients taking cART, which is a treatment that lowers viral replication. All of these observations indicate that reduced expression of GABAergic markers is not driven directly by the amount of replicating HIV-1 in the CNS or systemically. The lack of linkage with replicating HIV-1 as demonstrated herein is striking because it runs contrary to many conclusions suggested by prior work done using far fewer subjects (Masliah et al. 1995). Our conclusion regarding the lack of a direct role of HIV-1, while potentially counterintuitive and at-odds with prior suggestions draws support from the large patient sample. Our conclusion also is consistent with recent data showing that the neurovirological correlation between brain viral replication and HAND is not significant in most patients taking cART (Gelman et al. 2013).

\section{Neuroimmune Responses were Related to Abnormal Expression of GABAergic Markers}

This and other studies show that several aspects pertaining to the neurobiology of HIV infection in humans do not appear to be controlled directly by HIV replication in the CNS (Gelman et al. 2012a; Gelman et al. 2013). Indeed, there is abundant evidence that host immune responses in the CNS may play a critical role in driving HAND in a manner that is partly independent of HIV replication (Glass et al. 1995). Consistent with that, we found that low GABAergic transcripts were significantly correlated with higher neuroimmune type markers in the brain including interferon response genes. These findings agree with reports which showed that the host immune response in the CNS (e.g., activation of brain macrophages and microglia), but not the concentration of HIV RNA per $s e$, drove the development and progression of neurocognitive impairment (Glass et al. 1995; McArthur et al. 2010; Gelman et al. 2013). The fact that patients without encephalitis often had abnormal GABAergic transmission remains unexplained, yet is consistent with the fact that HAND also is highly prevalent in patients without encephalitis.

\section{Substance use History was not Related to Abnormal GABAergic Markers Expression}

Drug addiction was common in this HIV-infected patient cohort and could have influenced synaptic transmission; 89 out of 204 HIV-infected patients with self-reports had current or past history of substance abuse. That rate is comparable generally to other HIV-infected populations in which lifetime prevalence of any substance use ranges from 23 to $56 \%$ (Klinkenberg et al. 2004). In our cohort GABAergic expression was low in subjects with and without a history of drug dependence, and the two groups of subjects were not statistically different from each other. This result was somewhat unexpected because substance abuse often does result in the altered GABAergic neurotransmission. Altered expression of GABAergic mRNAs and proteins were reported for cocaine (Enoch et al. 2012), nicotine (Satta et al. 2008), methamphetamine (Zhang et al. 2006; Anneken et al. 2013), and morphine use (Sultana et al. 2010). These discrepancies are likely to be related to differences in experimental designs, as studies of substance abuse predominantly utilized animal models and 
acute dosing paradigms. The combined influence of substance abuse and HIV-1 on inhibitory system was studied in very few prior studies (Langford et al. 2003; Chana et al. 2006). It has been hypothesized that calbindin- and parvalbuminexpressing interneurons are selectively vulnerable to neurodegeneration in methamphetamine-using patients with HIVE. We suggest that the reason for our sharply contrasting conclusion (that these neurons do not undergo degeneration) is that we used multiple markers of inhibitory neurons to illustrate the lack of cell dropout. Other differences between this and previous studies include their use of smaller cohorts with highly restrictive inclusion and exclusion criteria, and the inherent differences between using postmortem human brain tissue versus animal models.

\section{The Neurovascular Unit is Linked to Abnormal Expression of GABAergic Markers}

Brain gene array data have suggested that patients with HAND who are taking cART, but do not have high viral replication rates in the CNS or encephalitis, might harbor a disturbance in the neurovascular unit (NVU) (Gelman et al. 2012a). The components of the NVU include endothelial cells, astrocytic end feet, nerve endings and accessory cells that include pericytes, perivascular microglial cells, and macrophages (Ballabh et al. 2004). It is notable that the most significant association of reduced expression of GABAergic markers that we observed was with higher expression of the brain endothelial cell markers. Since the endothelial cells are in direct contact with blood plasma, our results suggest that changes in GABAergic transmission may be linked via the NVU to systemic changes in virally suppressed patients, such as persistent inflammation (Gelman 2015). One possible scenario is that increased expression of cell adhesion molecules and endothelial markers (e.g., VCAM-1, ICAM-1, PECAM1, and von Willebrand factor) facilitates the transmigration of infected immune cells across the BBB (Eugenin et al. 2006). Increased concentrations of inflammatory molecules and viral proteins in the brain tissue often result in activated astroglia, disrupted glutamate / glutamine cycle and lower rate of GADs expression (Janda et al. 2011).

\section{Loss of GABAergic Immunostaining Does not Reflect Neurodegeneration}

Studies conducted before the era of cART suggested that HAND is a classical neurodegenerative disease that produces the loss of nonviable inhibitory interneurons. The validity of this suggestion as it pertains to cART-era brain specimens with HAND remains doubtful (Gelman and Moore 2011; Gelman 2015). This concept also has been challenged in patients with schizophrenia who have GABAergic disturbances (Benes et al. 1991). Our observations indicate that loss of
GABAergic mRNAs and protein occurs in viable inhibitory interneurons. DLPFC specimens from HIV-infected patients generally had decreased GAD67 staining intensity across the cortical lamina, and the affected interneurons still expressed calretinin, parvalbumin or somatostatin, which are alternative markers expressed by GABAergic neurons. The affected neuronal profiles had the morphological characteristics of fully viable interneurons with normally proportioned cell bodies and nuclear profiles that were not suggestive of necrosis or apoptosis. Our results offer little or no support for the hypothesis that the abnormal GABAergic neural transmission in HIV infected brain specimens is caused by classical neurodegeneration. The data suggest instead that transcription of genes is down-regulated, or that the stability of mRNAs of rate limiting enzymes is lost. Such changes lie within the range of changes that can drive synaptic plasticity and accommodation to stress (Gelman et al. 2012b). Our suggested interpretation is compatible with results from patients with schizophrenia, mood disorders, and Parkinson's disease, in whom neocortical GAD1 mRNA is abnormally expressed and loss of inhibitory neurons is not observed (Akbarian et al. 1995; Volk et al. 2000; Lanoue et al. 2010). Our results also agree with a report showing that parvalbumin-expressing interneurons did not drop out in the neocortex of patients with HIVE (Masliah et al. 1992). Taken altogether, the GABAergic disturbance in HIV infected patients probably reflects synaptic plasticity and accommodation instead of classical neurodegeneration of a subpopulation of cells.

\section{Reduced Expression of GABAergic Markers is Linked to Worse Cognitive Performance in HIV-1}

Down-regulated levels of GAD1 mRNA and GAD67 protein are characteristic features of various neurocognitive and psychiatric disorders, including autism, bipolar disorder, epilepsy, and schizophrenia (Akbarian et al. 1995; Volk et al. 2000; Thompson et al. 2009). This study found that HIV-infected patients with and without neurocognitive impairment both had lower GABAergic mRNAs, but were not significantly different when compared to each other. Moreover, we did not find significant difference between GABAergic mRNAs concentrations in groups with various degrees of cognitive impairment. Thus, abnormal GABAergic transmission per se is not a promising candidate biomarker for the nosological diagnosis of HAND. We did find, however, that all three GABAergic mRNAs were correlated specifically with worse performance on verbal fluency tasking, which also is abnormal in patients with frontal lobe trauma, Parkinson's disease, and Huntington's disease (Millikin et al. 2004). Verbal fluency tasking has two distinct functional components - phonemic (letter) and semantic (category) fluency, each consisting of two subtasks - clustering and switching. More than $40 \%$ of cases of impaired verbal fluency in HIV-infected patients have 
been linked to switching impairment (Millikin et al. 2004). Voxel-based lesion symptom mapping shows that phonemic switching is driven by the frontal lobes, whereas semantic switching relies on medial temporal lobes (Baldo et al. 2006). In HAND both semantic and phonemic fluency deficits are of similar magnitude, which suggests that frontal and temporal lobe functions both are involved. A key niche for future study is whether abnormal GABAergic transmission in the temporal lobes disproportionately impacts worse performance on tests of semantic switching.

\section{Abnormal Expression of GABAergic Markers is Interrelated with Other Neurotransmitter Transmission Systems}

Using the same cohort of HIV infected patients, it was shown previously that failure to suppress the expression of dopamine receptor type 2 long isoform mRNA (DRD2L) in the DLPFC was associated with worse neurocognitive performance (Gelman et al. 2012b). The implied interpretation is that lower expression of $D R D 2 L$ is a beneficial accommodation driven by higher presynaptic tone. The same report showed that enkephalinergic neural transmission also undergoes an accommodative type of decrease in many HIV infected brains. Both of those changes illustrate that synaptic plasticity could be central to the general mechanism of HAND. Abnormal expression of GABAergic genes was linked to changes associated with higher dopaminergic tone and lower enkephalinergic synaptic tone. These neurotransmitter systems interactions often could be observed histologically in tissue sections, as in our finding of higher expression of DRD2 in the interneurons lacking GAD67 immunoreactivity. These observations in HIV infected patients are consistent with reports from uninfected people that DRD2 is mostly expressed by large parvalbumin-expressing interneurons and small pyramidal neurons (Khan et al. 1998). Mechanistic studies indicate that dopamine stimulation of DRD2 and overexpression of DRD2 decreases GABA synthesis in inhibitory interneurons (Seamans et al. 2001). In turn, it is suggested that the lower GABA inhibitory currents lead to a shift toward excitation in local inhibitory microcircuits, which results in uncontrolled spread of activation and a decreased signal-tonoise ratio. At the behavioral level, the clinical manifestations of inhibitory circuit dysfunction include impaired working memory, planning and executive functions, slowed thinking, and word finding difficulties. All these features are characteristic neuropsychological findings in patients with schizophrenia and HAND (Seamans and Yang 2004; Iudicello et al. 2007; Woods et al. 2009). Thus, higher dopaminergic tone is one potential mechanism that could diminish GABAergic transmission in HIV-infected patients. While the differing types of neurotransmitter systems exhibit substantial interrelationships to each other, they do not necessarily drive the same functional deficit. For example, the GABAergic anomaly in DLPFC relates primarily to worse performance on tasks of verbal fluency (Table 1, discussed above), whereas defective dopaminergic tone in the DLPFC (Gelman et al. 2012b) was related to a broader spectrum of neurocognitive dysfunction (Seamans and Yang 2004). In contrast to those systems, abnormal enkephalinergic transmission (low PENK mRNA) showed little relationship to any of the neurocognitive functions that were examined. A highly complex picture emerges that involves shifting of multiple interconnected neurotransmitter systems, each having implications regarding particular aspects of abnormal neurocognitive function in HAND.

In sum, this neurochemical survey shows that GABAergic markers are abnormally low in the frontal neocortex of a substantial proportion of HIV infected patients. The differences were likely to have been regulated transcriptionally versus being the results of pathological neurodegeneration. Worse performance on tasks of verbal fluency was related significantly to lower frontocortical GABAergic marker expression, but other types of tasking were not related to it. Neither brain HIV replication nor encephalitis were significantly associated with GABAergic abnormalities. Brain markers associated with activated neuroimmunity and heightened endothelial cell activity both were linked significantly to low GABAergic marker expression, which suggests involvement of systemic immunity and the NVU.

Grant Support This work is supported by NIH grants R01NS072005, R01MH101017, R01MH79886. Human specimens and data were obtained from the National NeuroAIDS Tissue Consortium under the following U24 cooperative agreements: Texas NeuroAIDS Research Center: U24MH100930; California NeuroAIDS Tissue Network: U24MH100928; National Neurological AIDS Bank: U24MH100929; Manhattan HIV Brain Bank: U24MH100931; NNTC Data Coordinating Center: U24MH100925.

\section{Compliance with Ethical Standards}

Conflict of Interest The authors declare that they have no conflict of interest.

Open Access This article is distributed under the terms of the Creative Commons Attribution 4.0 International License (http:// creativecommons.org/licenses/by/4.0/), which permits unrestricted use, distribution, and reproduction in any medium, provided you give appropriate credit to the original author(s) and the source, provide a link to the Creative Commons license, and indicate if changes were made.

\section{References}

Akbarian S, Kim JJ, Potkin SG, Hagman JO, Tafazzoli A, Bunney WE, Jones EG (1995) Gene expression for glutamic acid decarboxylase is reduced without loss of neurons in prefrontal cortex of schizophrenics. Arch Gen Psychiatry 52:258-266

Ances BM, Vaida F, Cherner M, Yeh MJ, Liang CL, Gardner C, Grant I, Ellis RJ, Buxton RB, HIV Neurobehavioral Research Center 
(HNRC) Group (2011) HIV and chronic methamphetamine dependence affect cerebral blood flow. J Neuroimmune Pharmacol 6:409419

Anneken JH, Cunningham JI, Collins SA, Yamamoto BK, Gudelsky GA (2013) MDMA increases glutamate release and reduces parvalbuminpositive GABAergic cells in the dorsal hippocampus of the rat: role of cyclooxygenase. J Neuroimmune Pharmacol 8:58-65

Baldo JV, Schwartz S, Wilkins D, Dronkers NF (2006) Role of frontal versus temporal cortex in verbal fluency as revealed by voxel-based lesion symptom mapping. J Int Neuropsychol Soc 12:896-900

Ballabh P, Braun A, Nedergaard M (2004) The blood-brain barrier: an overview: structure, regulation, and clinical implications. Neurobiol Dis $16: 1-13$

Benes FM, McSparren J, Bird ED, San Giovanni JP, Vincent SL (1991) Deficits in small interneurons in prefrontal and cingulate cortices of schizophrenic and schizoaffective patients. Arch Gen Psychiatry 48(11):996-1001

Bennett MV, Zukin RS (2004) Electrical coupling and neuronal synchronization in the Mammalian brain. Neuron 41:495-511

Budka H (1991) Neuropathology of human immunodeficiency virus infection. Brain Pathol 1:163-175

Chana G, Everall IP, Crews L, Langford D, Adame A, Grant I, Cherner $\mathrm{M}$, and others (2006) Cognitive deficits and degeneration of interneurons in HIV+ Methamphetamine users. Neurology 67, no. 8

Enoch MA, Zhou Z, Kimura M, Mash DC, Yuan Q, Goldman D (2012) GABAergic gene expression in postmortem hippocampus from alcoholics and cocaine addicts; corresponding findings in alcoholnaïve $\mathrm{P}$ and NP rats. PLoS One 7, e29369

Ernst T, Jiang CS, Nakama H, Buchthal S, Chang L (2010) Lower brain glutamate is associated with cognitive deficits in HIV patients: a new mechanism for HIV-associated neurocognitive disorder. J Magn Reson Imaging 32:1045-1053

Eugenin EA, Gamss R, Buckner C, Buono D, Klein RS, Schoenbaum EE, Calderon TM, Berman JW (2006) Shedding of PECAM-1 during HIV infection: a potential role for soluble PECAM-1 in the pathogenesis of NeuroAIDS. J Leukoc Biol 79:444-452

Fergus A, Lee KS (1997) GABAergic regulation of cerebral microvascular tone in the rat. J Cerebral Blood Flow Metabolism: Off J Int Soc Cerebral Blood Flow Metabolism 17, no. 9.

Fox L, Alford M, Achim C, Mallory M, Masliah E (1997) Neurodegeneration of somatostatin-immunoreactive neurons in HIV encephalitis. J Neuropathol Exp Neurol 56:360-368

Gelman BB (2015) Neuropathology of HAND with suppressive antiretroviral therapy: encephalitis and neurodegeneration reconsidered. Curr HIV/AIDS Rep 12(2):272-9

Gelman BB, Moore DJ (2011) The neuropathology of HIV-1. In Howard E. Gendelman, Igor Grant, Ian Paul Everall, Howard S. Fox, Harris A. Gelbard, Stuart A. Lipton, Susan Swindells (Eds.) The Neurology of AIDS 3 ed. (pp 518-536). Oxford University Press

Gelman BB, Nguyen TP (2010) Synaptic proteins linked to HIV-1 infection and immunoproteasome induction: proteomic analysis of human synaptosomes. J Neuroimmune Pharmacol 5:92-102

Gelman BB, Soukup VM, Schuenke KW, Keherly MJ, Holzer C, Richey FJ, Lahart CJ (2004) Acquired neuronal channelopathies in HIVassociated dementia. J Neuroimmunol 157:111-119

Gelman BB, Spencer JA, Holzer CE, Soukup VM (2006) Abnormal striatal dopaminergic synapses in national NeuroAIDS tissue consortium subjects with HIV encephalitis. J Neuroimmune Pharmacol $1: 410-420$

Gelman BB, Chen T, Lisinicchia JG, Soukup VM, Carmical JR, Starkey JM, Masliah E, Commins DL, Brandt D, Grant I, Singer EJ, Levine AJ, Miller J, Winkler JM, Fox HS, Luxon BA, Morgello S, National NeuroAIDS Tissue Consortium (2012a) The national NeuroAIDS tissue consortium brain gene array: two types of HIV-associated neurocognitive impairment. PLoS One 7, e46178
Gelman BB, Lisinicchia JG, Chen T, Johnson KM, Jennings K, Freeman DH, Soukup VM (2012b) Prefrontal dopaminergic and enkephalinergic synaptic accommodation in HIV-associated neurocognitive disorders and encephalitis. J Neuroimmune Pharmacol

Gelman BB, Lisinicchia JG, Morgello S, Masliah E, Commins D, Achim CL, Fox HS, Kolson DL, Grant I, Singer E (2013) Neurovirological correlation with HIV-associated neurocognitive disorders and encephalitis in a HAART-era cohort. JAIDS J Acquired Immune Deficiency Syndromes 62:487-495

Glass JD, Fedor H, Wesselingh SL, McArthur JC (1995) Immunocytochemical quantitation of human immunodeficiency virus in the brain: correlations with dementia. Ann Neurol 38:755-762

Hashimoto T, Arion D, Unger T, Maldonado-Avilés JG, Morris HM, Volk DW, Mirnics K, Lewis DA (2008) Alterations in GABA-related transcriptome in the dorsolateral prefrontal cortex of subjects with schizophrenia. Mol Psychiatry 13:147-161

Hestrin S, Galarreta M (2005) Electrical synapses define networks of neocortical GABAergic neurons. Trends Neurosci 28:304-309

Iudicello, JE, Woods SP, Parsons TD, Moran LM, Carey CL, Grant I (2007) Verbal fluency in HIV infection: a meta-analytic review. J Int Neuropsychol Soc 13, no. 1: doi:10.1017/S1355617707070221

Janda E, Visalli V, Colica C, Aprigliano S, Musolino V, Vadalà N, Muscoli C, and others (2011) The protective effect of tianeptine on Gp120-induced apoptosis in astroglial cells: role of GS and NOS, and NF-kB Suppression. "British J Pharmacology 164, no. 6

Khan ZU, Gutiérrez A, Martín R, Peñafiel A, Rivera A, De La Calle A (1998) Differential regional and cellular distribution of dopamine D2-like receptors: an immunocytochemical study of subtypespecific antibodies in rat and human brain. J Comp Neurol 402(3): $353-71$

Klinkenberg WD, Sacks S, HIV/AIDS Treatment Adherence, Health Outcomes and Cost Study Group (2004) Mental disorders and drug abuse in persons living with HIV/AIDS. AIDS Care 16(Suppl 1): S22-S42

Kocharyan A, Fernandes P, Tong XK, Vaucher E, Hamel E (2008) Specific subtypes of cortical GABA interneurons contribute to the neurovascular coupling response to basal forebrain stimulation. $\mathrm{J}$ Cereb Blood Flow Metab 28:221-231

Koutsilieri E, ter Meulen V, Riederer P (2001) Neurotransmission in HIV associated dementia: a short review. J Neural Transm 108:767-775

Langford D, Adame A, Grigorian A, Grant I, McCutchan JA, Ellis RJ, Marcotte TD, Masliah E, HIV Neurobehavioral Research Center Group (2003) Patterns of selective neuronal damage in methamphetamine-user AIDS patients. J Acquir Immune Defic Syndr 34:467-474

Lanoue AC, Dumitriu A, Myers RH, Soghomonian JJ (2010) Decreased glutamic acid decarboxylase MRNA expression in prefrontal cortex in parkinson's disease. Experimental Neurology 226, no. 1

Markram H, Toledo-Rodrigez M, Wang Y, Gupta A, Sillberger G, Wu C (2004) Interneurons of the cortical inhibitory system. Nat Rev Neurosci 5:793-807

Masliah E, Ge N, Achim CL, Hansen LA, Wiley CA (1992) Selective neuronal vulnerability in HIV encephalitis. J Neuropathol Exp Neurol 51:585-593

Masliah E, Ge N, Achim CL, Wiley CA (1995) Differential vulnerability of calbindin-immunoreactive neurons in HIV encephalitis. J Neuropathol Exp Neurol 54:350-357

Masliah E, Heaton RK, Marcotte TD, Ellis RJ, Wiley CA, Mallory M, Achim CL, McCutchan JA, Nelson JA, Atkinson JH, Grant I (1997) Dendritic injury is a pathological substrate for human immunodeficiency virus-related cognitive disorders. HNRC Group. The HIV Neurobehavioral Research Center. Ann Neurol 42:963-972

McArthur JC, Steiner J, Sacktor N, Nath A (2010) Human immunodeficiency virus-associated neurocognitive disorders: mind the gap. Ann Neurol 67:699-714 
Millikin CP, Trépanier LL, Rourke SB (2004) Verbal fluency component analysis in adults with HIV/AIDS. J Clin Exp Neuropsychol 26: 933-942

Morgello S, Gelman BB, Kozlowski PB, Vinters HV, Masliah E, Cornford M, Cavert W, Marra C, Grant I, Singer EJ (2001) The national NeuroAIDS tissue consortium: a new paradigm in brain banking with an emphasis on infectious disease. Neuropathol Appl Neurobiol 27:326-335

Murray MF (2003) Tryptophan depletion and HIV infection: a metabolic link to pathogenesis. Lancet Infect Dis 3:644-652

Nath A, Jones M, Booze M, Bell H, Mattson (2000) Neurotoxicity and dysfunction of dopaminergic systems associated with AIDS dementia. J Psychopharmacol 14:222-227

Nguyen TP, Soukup VM, Gelman BB (2010) Persistent hijacking of brain proteasomes in HIV-associated dementia. Am J Pathol 176:893-902

Northoff G, Walter M, Schulte RF, Beck J, Dydak U, Henning A, Boeker $\mathrm{H}$, Grimm S, Boesiger P (2007) GABA concentrations in the human anterior cingulate cortex predict negative BOLD responses in FMRI. Nature Neuroscience 10, no. 12

Pinto JG, Hornby KR, Jones DG, Murphy KM (2010) Developmental changes in GABAergic mechanisms in human visual cortex across the lifespan. Front Cell Neurosci 4:16

Prince DA, Wilder BJ (1967) Control mechanisms in cortical epileptogenic Foci* "Surround" inhibition. Arch Neurol 16:194

Robins LN, Wing J, Wittchen HU, Helzer JE, Babor TF, Burke J, Farmer A, Jablenski A, Pickens R, Regier DA (1988) The composite international diagnostic interview. An epidemiologic Instrument suitable for use in conjunction with different diagnostic systems and in different cultures. Arch Gen Psychiatry 45:1069-1077

Sanacora G, Saricicek A (2007) GABAergic contributions to the pathophysiology of depression and the mechanism of antidepressant action. CNS Neurological Disorders - Drug Targets 6:127-140

Satta R, Maloku E, Zhubi A, Pibiri F, Hajos M, Costa E, Guidotti A (2008) Nicotine decreases DNA methyltransferase 1 expression and glutamic acid decarboxylase 67 promoter methylation in GABAergic interneurons. Proc Natl Acad Sci U S A 105:1635616361

Seamans JK, Yang CR (2004) The principal features and mechanisms of dopamine modulation in the prefrontal cortex. Prog Neurobiol 74:158
Seamans JK, Gorelova N, Durstewitz D, Yang CR (2001) Bidirectional dopamine modulation of GABAergic inhibition in prefrontal cortical pyramidal neurons. J Neurosci 21:3628-3638

Seney ML, Chang LC, Oh H, Wang X, Tseng GC, Lewis DA, Sibille E (2013) The role of genetic sex in affect regulation and expression of GABA-related genes across species. Front Psychiatry 4:104

Soghomonian JJ, Martin DL (1998) Two isoforms of glutamate decarboxylase: why? Trends Pharmacol Sci 19:500-505

Strazza M, Pirrone V, Wigdahl B, Nonnemacher MR (2011) Breaking down the barrier: the effects of HIV-1 on the blood-brain barrier. Brain Res 1399:96-115

Sultana S, Li H, Puche A, Jones O, Bryant JL, Royal W (2010) Quantitation of parvalbumin + neurons and human immunodeficiency virus type 1 (HIV-1) regulatory gene expression in the HIV-1 transgenic rat: effects of vitamin A deficiency and morphine. J Neurovirol 16:33-40

Thompson M, Weickert CS, Wyatt E, Webster MJ (2009) Decreased glutamic acid decarboxylase(67) mRNA expression in multiple brain areas of patients with schizophrenia and mood disorders. J Psychiatr Res 43:970-977

Towgood KJ, Pitkanen M, Kulasegaram R, Fradera A, Soni S, Sibtain N, Reed LJ, Bradbeer C, Barker GJ, Dunn JT, Zelaya F, Kopelman MD (2012) Regional cerebral blood flow and FDG uptake in asymptomatic HIV-1 men. Hum Brain Mapp.

Volk DW, Austin MC, Pierri JN, Sampson AR, Lewis DA (2000) Decreased glutamic acid decarboxylase67 messenger RNA expression in a subset of prefrontal cortical gamma-aminobutyric acid neurons in subjects with schizophrenia. Arch Gen Psychiatry 57: 237-245

Woods SP, Rippeth JD, Frol AB, Levy JK, Ryan E, Soukup VM, Hinkin CH, Lazzaretto D, Cherner M, Marcotte TD, Gelman BB, Morgello S, Singer EJ, Grant I, Heaton RK (2004) Interrater reliability of clinical ratings and neurocognitive diagnoses in HIV. J Clin Exp Neuropsychol 26:759-778

Woods SP, Moore DJ, Weber E, Grant I (2009) Cognitive neuropsychology of HIV-associated neurocognitive disorders. Neuropsychol Rev 19:152-168

Zhang X, Lee TH, Xiong X, Chen Q, Davidson C, Wetsel WC, Ellinwood EH (2006) Methamphetamine induces long-term changes in GABAA receptor alpha2 subunit and GAD67 expression. Biochem Biophys Res Commun 351:300-305 